\title{
Creativity, ambiguous figures, and academic preference
}

\author{
Martin J Doherty, Samantha Mair \\ Department of Psychology, University of Stirling, Stirling FK9 4LA, UK; \\ e-mail: m.j.doherty@stir.ac.uk \\ Received 1 August 2012, in revised form 17 September 2012
}

\begin{abstract}
Research suggests that ambiguous figure reversal is associated with creativity, but current evidence relies on subjective self-report that is difficult to quantify (Wiseman, Watt, Gilhooly, Georgiou, 2011 British Journal of Psychology 102 615-622). Using quantifiable measures of both phenomena we confirm this claim. We also find that participants studying science experience much more frequent reversal - a novel and intriguing finding.
\end{abstract}

Keywords: ambiguous figure reversal, creativity, academic preference

\section{Introduction}

Recently Wiseman et al (2011) presented evidence for a relationship between creativity and ease of reversal of an ambiguous figure. As they note, this conclusion is limited by their self-report measure: participants were shown an ambiguous figure and asked to rate how easy they found it to see the opposite interpretation. Most participants rated this as easy or very easy (95\% in study $1,85 \%$ in study 2 ), suggesting that they had no baseline against which to judge their relative reversal ability. Individual differences therefore might reflect factors like self-confidence rather than reversal ability. We replicate the finding using quantifiable measures of reversal. We also extend the work by using more than one type of ambiguous figure. Ambiguous figures fall into three distinct categories, in which reversal is of figure and ground, content, or perspective (Long and Toppino 2004). See figure 1 for exemplars.
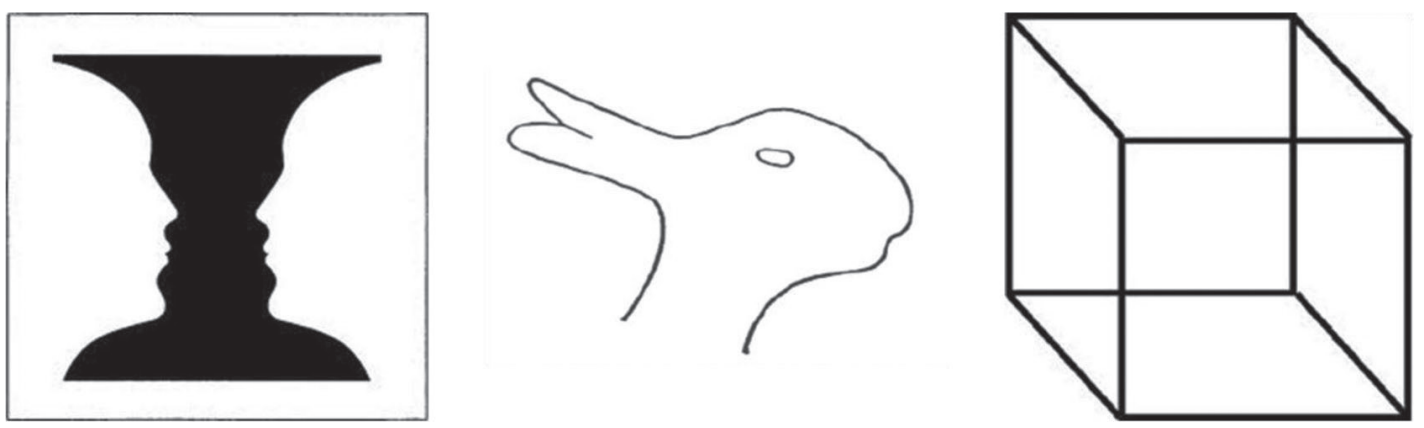

Figure 1. The three ambiguous figures used in this study.

\section{Method}

\subsection{Participants}

Participants were thirty-eight secondary school students (nineteen boys) between the ages of 16 years and 18 years 4 months (mean age 17 years 2 months, $S D=6$ months). Participants studied between 3 and 5 subjects, classified as sciences (physics, chemistry, biology, and maths) or arts and social sciences (English, languages, music, history, media studies, etc). Sciences comprised $36 \%$ of subjects. An academic preference score from +1 (all sciences) to -1 (all arts and social sciences) was constructed for each participant by adding +1 for each science and -1 for each non-science subject divided by the total number of subjects studied (mean $=-0.278, \mathrm{SD}=0.53$ ). 


\subsection{Ambiguous (figures task)}

This task included three ambiguous figures shown in figure 1. Images were approximately $12 \mathrm{~cm} \times 12 \mathrm{~cm}$, presented separately. Participants were asked to look at the image for $1 \mathrm{~min}$, and to inform the experimenter when they noticed a change in their interpretation, making a dash in the test booklet for each subsequent reversal.

\subsection{Pattern meanings test (Wallach and Kogan, 1965)}

This test comprises eight abstract patterns (figure 2) presented individually on $20 \times 14 \mathrm{~cm}$ laminated cards. Participants were asked to write down "all of the things you think it could be, or that it reminds you of". One point was given for each novel interpretation, excluding variations on the same object (eg 'glasses' and 'sunglasses').
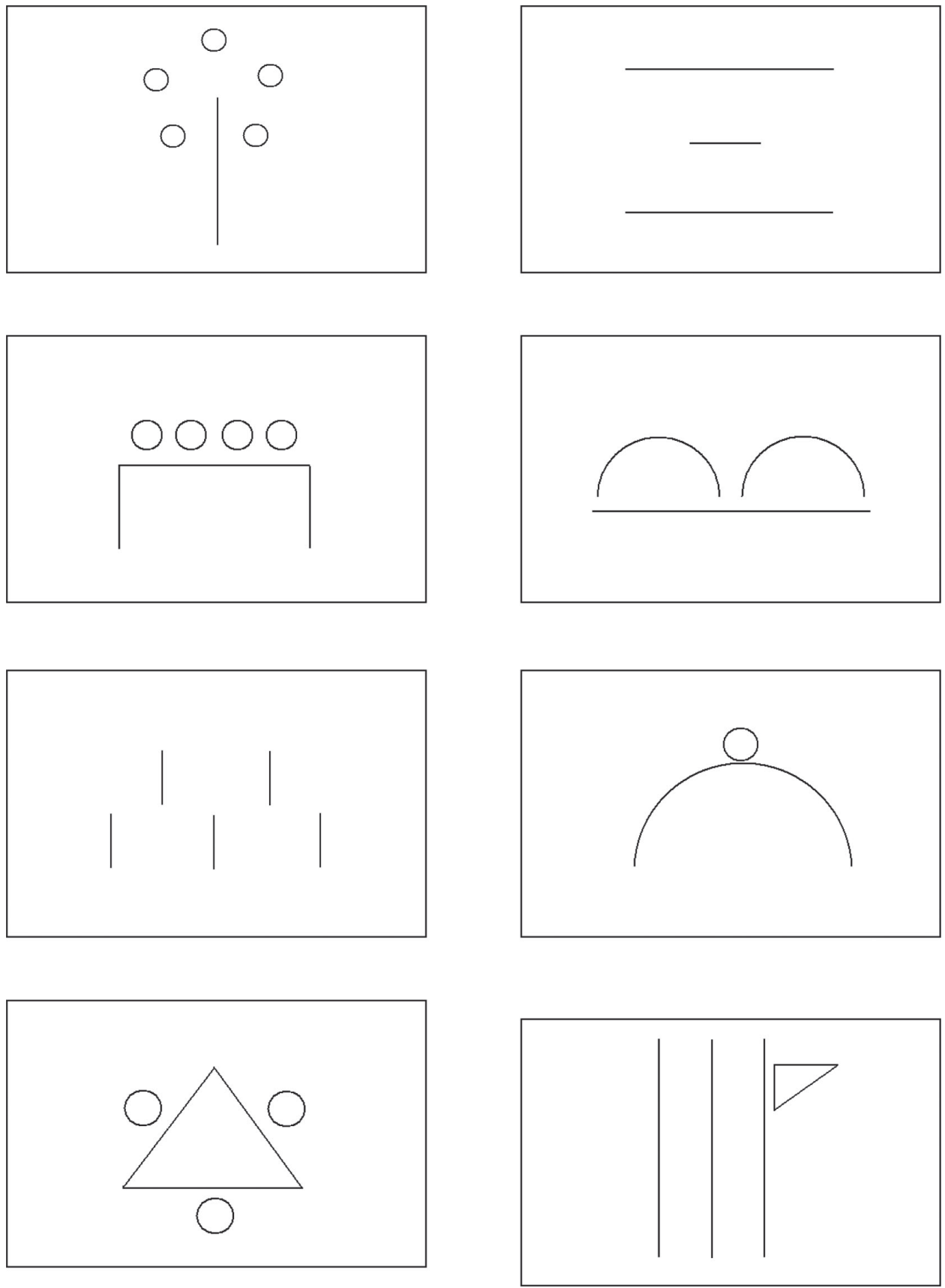

Figure 2. Stimuli for the pattern meanings test, adapted from Wallach and Kogan (1965). 
The second aim is to examine how both factors relate to choice of academic discipline, on the intuitive hypothesis that studying science requires or fosters the ability to switch between alternative interpretations of a stimulus. Cognitive or perceptual styles have been found to co-vary with academic discipline in previous research. Phillips et al (2004) found that social scientists were more susceptible to the Ebbinghaus visual illusion than mathematical scientists, an effect that extends to other cultures (Doherty et al 2008).

We measured the time it took participants to experience initial reversal of ambiguous figures and the frequency of reversal during $1 \mathrm{~min}$. Creativity was measured with Wallach and Kogan's (1965) pattern meanings test. Participants were shown abstract patterns and asked what they could be (figure 2). This is analogous to the phenomenology of reversal, in which a stimulus can suddenly be seen in a new way.

\section{Results}

\subsection{Pattern meanings task}

Participants produced roughly two alternative interpretations for each pattern, summed to produce a creativity score (roughly normally distributed, range 2 to 32 , mean $=16.7, \mathrm{SD}=6.58$ ).

\subsection{Ambiguous figures task}

Mean times to first reversal and the frequencies of reversal for the three figures are shown in table 1. Participants took longer to initially reverse the Necker cube than the vase-faces (Friedman, $Z=2.67, p<0.01$ ); no other differences were significant. Frequencies of reversal did not differ between figures $\left(\chi^{2}=1.42, p=0.49\right)$, and were substantially correlated (table 2 ).

Table 1. Mean time to first reversal and frequency of reversal for the three ambiguous figures. Standard deviations are shown in parentheses.

\begin{tabular}{lccrr}
\hline Ambiguous figures & \multicolumn{2}{c}{ Time to first reversal/s } & \multicolumn{2}{c}{ Number of reversals per minute } \\
\hline Vase-faces & 12.4 & $(19.0)$ & 13.9 & $(17.5)$ \\
Duck-rabbit & 14.7 & $(18.2)$ & 12.3 & $(15.6)$ \\
Necker cube & 16.9 & $(16.6)$ & 8.2 & $(9.9)$ \\
\hline
\end{tabular}

Table 2. Correlations between frequency of reversal for the three ambiguous figures, creativity, and academic preference.

\begin{tabular}{|c|c|c|c|c|}
\hline & \multicolumn{3}{|l|}{ Frequency } & \multirow[t]{2}{*}{ Academic preference } \\
\hline & vase-faces & duck-rabbit & Necker cube & \\
\hline Creativity & $0.39 *$ & 0.20 & $0.49 * *$ & 0.15 \\
\hline \multicolumn{5}{|l|}{ Frequency } \\
\hline vase-faces & & $0.78^{* * *}$ & $0.50 * * *$ & $0.40^{*}$ \\
\hline duck-rabbit & & & $0.35^{*}$ & $0.42 * *$ \\
\hline Necker cube & & & & 0.14 \\
\hline
\end{tabular}

\subsection{Comparison of measures}

Creativity scores were significantly related to overall frequency of reversal $\left(r_{\mathrm{s}}=0.42\right.$, $p<0.01)$. There was also a trend towards a relationship between creativity scores and time to first reversal $\left(r_{\mathrm{s}}=-0.30, p=0.07\right)$. Both overall frequency of reversal and time to first reversal were significantly associated with academic preference $\left(r_{\mathrm{s}}=0.39\right.$ and $r_{\mathrm{s}}=-0.36$, respectively, both $p \mathrm{~s}<0.05$ ). 
Table 2 shows relationships between creativity, academic preference, and frequency of reversal of individual figures. The relationship between frequency and creativity scores is largely determined by the Necker cube and vase-faces, whereas that between frequency and academic preference by the vase-faces and duck-rabbit.

Figure 3 illustrates the relationship between reversal frequency and creativity when participants are split by the mean into high and low creativity groups (producing between 17 and 32, or 16 or fewer interpretations on the pattern meanings test, respectively). More creative participants reversed each figure more frequently.

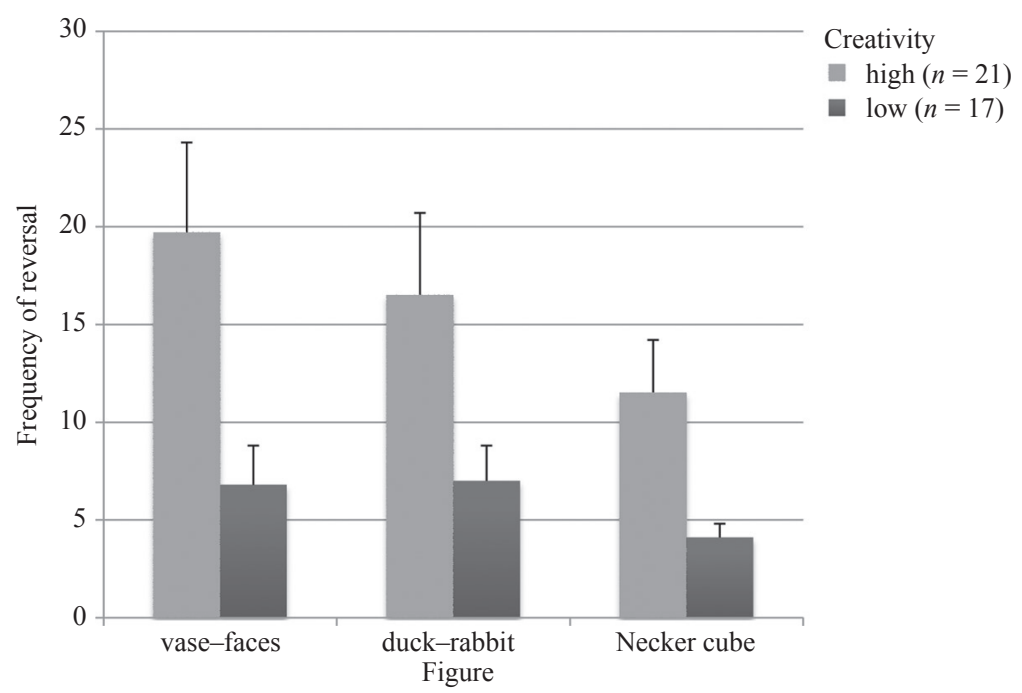

Figure 3. Frequency of reversal for participants with high and low creativity scores.

Figure 4 illustrates the relationship between reversal frequency and academic preference when participants are grouped into science and arts/social science groups (preference scores between 0 and 1 , or less than 0 , respectively). Participants with a preference for science subjects reversed figures more frequently than participants preferring arts or social sciences.

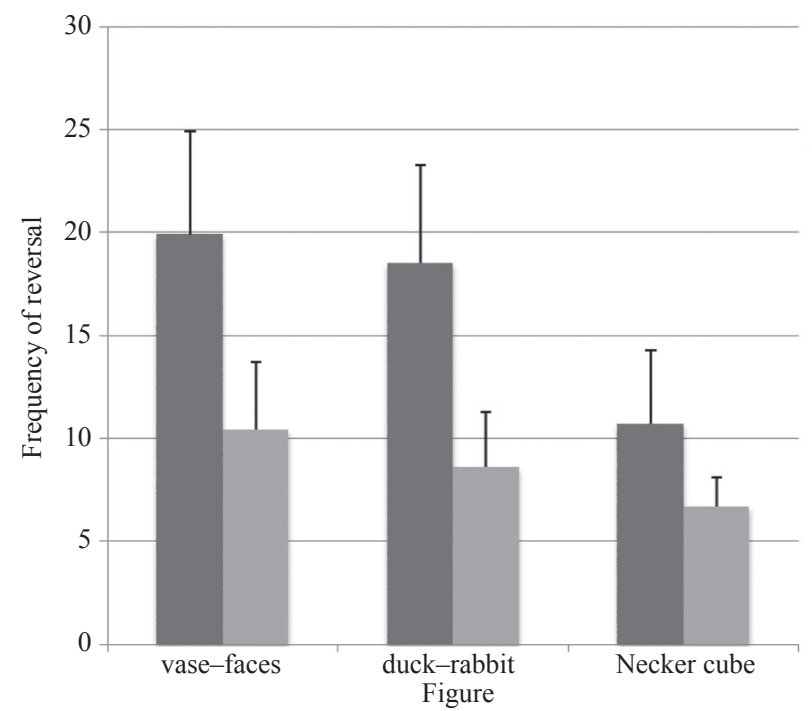

science $(n=14)$

arts/social science $(n=24)$

Figure 4. Frequency of reversal according to participants' academic preferences. 


\section{Discussion}

We examined Wiseman et al's (2011) claim that creativity and ambiguous figure reversal are related, substituting their subjective rating of reversal difficulty with more quantifiable measures. Frequency of reversal was substantially related to our measure of creativity. We therefore conclude that the claim is broadly correct.

Most surprisingly, participants' academic preferences were related to reversal. Faster initial and more frequent reversals were associated with a preference for science. Whether this is fostered by studying science or influences an individual's initial choice to study it warrants further investigation.

The differential pattern of relations between variables conforms to whether a figure involves reversal of perspective (Necker cube), of meaning or content (duck-rabbit), or reversal of both (vase-faces). The relationship between reversal and creativity is strongest for the two figures involving reversal of perspective. The relationship between reversal and academic preference is strongest for the figures involving reversal of meaning.

The creativity relationships may be due to our creativity measure, which requires imposing a three-dimensional imagined shape onto a series of two-dimensional lines, followed by a different shape for each subsequent interpretation. This is intuitively similar to Neckercube reversal. On the other hand, Wiseman et al (2011) measured creativity by asking participants to think of alternative uses for a brick or a paperclip (Guilford et al 1978). This can be thought of as seeing a new meaning (use) for objects, and performance was associated with their measure of ease of reversal of Jastrow's classic duck-rabbit figure.

This allows a prediction: the alternative uses task should also associate with a preference for science. The ability to see new meanings in stimuli is certainly a key skill in scientific reasoning, and may be why academic preference associates most highly with reversal of meaning.

Future studies must go beyond such broad intuitions to examine how creativity and academic preference relate to other factors that influence reversal. Recent developmental research suggests mental imagery and executive inhibition are key component skills (Wimmer and Doherty 2011). The three types of figure may draw differentially on each skill. Clearly our creativity measure heavily involves mental imagery. Plausibly scientific reasoning requires good inhibitory ability, but to our knowledge this has yet to be studied.

\section{References}

Doherty M J, Tsuji H, Phillips W A, 2008 "The context-sensitivity of visual size perception varies across cultures" Perception 37 1426-1433

Guilford J P, Christensen P R, Merrifield P R, Wilson R C, 1978 Alternate Uses: Manual of Instructions and Interpretations (Orange, CA : Sheridan Psychological Services)

Long G M, Toppino T C, 2004 "Enduring interest in perceptual ambiguity: alternating views of reversible figures" Psychological Bulletin 130 748-768

Phillips W A, Chapman K L S, Berry P D, 2004 "Size perception is less context-sensitive in males" Perception 33 79-86

Wallach M A, Kogan N, 1965 Modes of Thinking in Young Children: A Study of the CreativityIntelligence Distinction (New York: Holt, Rinehart, and Winston)

Wimmer M C, Doherty M J, 2011 "The development of ambiguous figure perception" Monographs of the Society for Research in Child Development 76(1) 1-130

Wiseman R, Watt C, Gilhooly K, Georgiou G, 2011 "Creativity and ease of ambiguous figure reversal" British Journal of Psychology 102 615-622 